\title{
Train tracking problem using a hybrid system model
}

\author{
Y. Wang, R. Luo, F. Cao \& B. Ning \\ State Key Laboratory of Rail Traffic Control and Safety, \\ Beijing Jiaotong University, China
}

\begin{abstract}
Tracking is an important problem in train operation control. A key requirement for this problem is an accurate knowledge of the train's position, velocity, and running mode. In this paper a hybrid system model of the train's movement is introduced, which, for the first time, gives a clear description of the uncertainties during the movement. Based on this hybrid model, a new hybrid estimation algorithm is proposed in order to achieve a more accurate estimation of the train's states, thereby improving the tracking performance. In the algorithm, the state transition probability matrix is dependent on the operation mode. Simulation results illustrate the good performance of the new estimation algorithm with the hybrid system model.
\end{abstract}

Keywords: hybrid system, automatic train operation, tracking, estimation.

\section{Introduction}

The automatic train operation system is one of the key sub-systems in trains. Accurate estimation of the train's velocity and position is the basis for the safety of the automatic train operation. With that, the train tracking problem becomes more and more important for obtaining an accurate estimation of the train's states. Hybrid estimation algorithms have been used in many target tracking applications, including air traffic surveillance $[1,2]$.

In this paper, a hybrid system model is proposed for modelling the train's dynamics. Four operation modes, power, speed holding, coast and braking, are modelled as the discrete states of the system, under which the train operates based on a continuous-time dynamic equation. Meanwhile, our model considers 
the stochastic factors due to the uncertainties in the train movement. Few literatures consider these, yet they have a great effect on the tracking problems.

Based on the hybrid model of the train's movement, a new hybrid estimation algorithm is proposed to track the train's movement and estimate the train's operation state. Interacting the Multiple Model (IMM) algorithm is a popular hybrid estimation algorithm based on multiple-model Kalman filters. It has been shown to give excellent performance with low computational cost in Blom and Bar-Shalom [5]. However, the IMM algorithm and other similar algorithms usually assume constant mode transition probabilities. The estimation algorithm presented in this paper has different mode transition probabilities corresponding to different modes, called the Mode-Dependent-Hybrid-Estimation (MDHE) algorithm. The simulation results show that the proposed algorithm achieves more accurate tracking and estimation performance compared with the IMM algorithm.

This paper is organized as follows: Sec. 2 introduces a stochastic linear hybrid system model of train dynamics. Sec. 3 proposes a corresponding hybrid estimation algorithm for the train tracking problem. In Sec. 4, the simulation illustrates the performance of the algorithm. Conclusions are presented in Sec. 5.

\section{Hybrid model of train movement}

A hybrid system is a system whose evolution is driven by both the continuous time and the discrete events. The dynamics of continuous components are described by the traditional differential/difference equations. Only when some conditions are satisfied, jumps of the system's state are triggered by discrete events. In the train control system, the train's states change continuously with time, such as velocity and position, which can be described by differential equations [3,6]. However, they will run into different modes triggered by discrete events, such as the switches between traction and brake. In the train's movement, there are four operation modes: power, speed-holding, coast and braking. Let $M=\{1,2,3,4\}$ correspond to these four discrete modes. It is assumed that $p \geq 0$ is the traction power applied at the wheels and $P$ is the maximum power, $q \geq 0$ is the braking force and $Q$ is the maximum braking force.

To describe the train dynamics in each mode, we define $x=[s, \dot{s}, \ddot{s}]^{T}$ as the continuous states vector, where $s$ denotes the train's position, $\dot{s}$ denotes velocity, and $\ddot{s}$ denotes acceleration. In the hybrid model of the train's movement, the uncertainty inherent in the train's motion is considered. The uncertainty is due to traction and braking ability, weight bearing, climate factors and so on, which is modelled by different white Gaussian noises with respect to different modes. Let $t_{k}=t_{0}+k T_{s}$ be the sampling time instant started from $t_{0}$, where $T_{s}$ is the sample interval, and $k=1,2, \ldots$. The train dynamics in each mode are described as follows. 


\subsection{Train dynamics}

1) Power Mode. The traction force is equal to the maximum power and the braking force is zero. The control in power mode is given by $p=P$ and $q=0$. In the power mode, we model the uncertainties as a white Gaussian noise. The train dynamics are described by

$$
x(k+1)=\left[\begin{array}{ccc}
1 & T_{s} & T_{s}^{2} / 2 \\
0 & 1 & T_{s} \\
0 & 0 & 1
\end{array}\right] x(k)+\left[\begin{array}{c}
T_{s}^{2} / 2 \\
T_{s} \\
1
\end{array}\right] \omega_{\text {Power }},
$$

where $\omega_{\text {Power }}$ is white Gaussian noise with mean zero and covariance:

$$
\xi_{\text {Power }}=E\left[\omega_{\text {Power }}^{2}\right]=0.05^{2}\left(\mathrm{~m} / \mathrm{s}^{2}\right)^{2}
$$

Different covariances are chosen for different modes by analyzing the train running conditions and moving data.

2) Hold Mode. If the train is running at a constant speed, we call this mode speed holding or simply hold. When the train is in this mode, the traction power changes with various resistances and braking force $q=0$. The model is given by

$$
x(k+1)=\left[\begin{array}{ccc}
1 & T_{s} & 0 \\
0 & 1 & 0 \\
0 & 0 & 0
\end{array}\right] x(k)+\left[\begin{array}{c}
T_{s}^{2} / 2 \\
T_{s} \\
1
\end{array}\right] \omega_{\text {Hold }},
$$

where $\varpi_{\text {Hold }}$ is white Gaussian noise with mean zero and covariance:

$$
\xi_{\text {Hold }}=E\left[\omega_{\text {Hold }}^{2}\right]=0.03^{2}\left(\mathrm{~m} / \mathrm{s}^{2}\right)^{2}
$$

3) Coast Mode. There is no power applied and no braking in coast mode, i.e. $p=0, q=0$. In the coast mode, the model is similar to that the model used in power mode.

$$
x(k+1)=\left[\begin{array}{ccc}
1 & T_{s} & T_{s}^{2} / 2 \\
0 & 1 & T_{s} \\
0 & 0 & 1
\end{array}\right] x(k)+\left[\begin{array}{c}
T_{s}^{2} / 2 \\
T_{s} \\
1
\end{array}\right] \omega_{\text {Coast }}
$$

The process noise in the Coast mode is

$$
\xi_{\text {coast }}=E\left[\omega_{\text {Coast }}^{2}\right]=0.01^{2}\left(\mathrm{~m} / \mathrm{s}^{2}\right)^{2}
$$

4) Braking Mode. In the Braking mode, the speed declines by full braking force, i.e. $p=0$ and $q=Q$. The dynamic model is as following:

$$
x(k+1)=\left[\begin{array}{ccc}
1 & T_{s} & T_{s}^{2} / 2 \\
0 & 1 & T_{s} \\
0 & 0 & 1
\end{array}\right] x(k)+\left[\begin{array}{c}
T_{s}^{2} / 2 \\
T_{s} \\
1
\end{array}\right] \omega_{\text {Braking }}
$$

The process noise $\omega_{\text {Braking }}$ is a white Gaussian noise with mean zero and covariance

$$
\xi_{\text {Braking }}=E\left[\omega_{\text {Braking }}^{2}\right]=0.05^{2}\left(\mathrm{~m} / \mathrm{s}^{2}\right)^{2}
$$




\subsection{Measurement model}

In train control systems, the measurement of train's speed and position are taken by the corresponding sensors. All measurements are subject to uncertainty due to the time delay and measurement disturbance. Thus, it can always be approximated by a linear model given by

$$
z(k)=\left[\begin{array}{lll}
1 & 0 & 0 \\
0 & 1 & 0
\end{array}\right] x(k)+\left[\begin{array}{l}
v_{s}(k) \\
v_{\dot{s}}(k)
\end{array}\right],
$$

where $v_{s}(k), v_{\dot{s}}(k)$ are Gaussian noise with mean zero and covariance:

$$
R=\left[\begin{array}{cc}
E\left[v_{s}^{2}\right] & 0 \\
0 & E\left[v_{\dot{s}}^{2}\right]
\end{array}\right]=\left[\begin{array}{cc}
0.1 & 0 \\
0 & 0.05
\end{array}\right]
$$

\section{Hybrid estimation algorithm for train tracking}

We rewrite the train dynamics as a stochastic linear hybrid model as:

$$
\begin{gathered}
x(k)=A_{m(k)} x(k-1)+D \omega_{m(k)}(k) \\
z(k)=C_{m(k)} x(k)+v_{m(k)}(k)
\end{gathered}
$$

Where $x(k) \in \mathrm{R}^{n}$ and $z(k) \in \mathrm{R}^{p}$ are continuous state and the measurement variables, respectively. $m(k) \in M=\{1,2,3,4\}$ is the discrete state at time $k$, corresponding to four different operation modes: Power, Hold, Coast, and Braking. The process noise $\omega_{m(k)}(k)$ and the measurement noise $v_{m(k)}(k)$ are uncorrelated Gaussian sequences with zero mean. We use $m(k)=j$ to denote the event that the system is in mode $j$ at time $k$, and $m(k-1)=i$ to denote the event that the system is in mode $i$ at time $k-1$. A continuous-state-dependent mode transition matrix is defined to describe the evolution of mode $m(k)$ :

$$
\begin{gathered}
\Pi(x(k-1))=\left\{\pi_{i j}(x(k-1))\right\}_{i, j=1,2,3,4} \\
\pi_{i j}(x(k-1)):=p[j \mid i, x(k-1)]
\end{gathered}
$$

for $i, j \in\{1,2,3,4\}$. It is worthy to note that in some linear hybrid estimation algorithms, such as IMM algorithm, the mode transition matrix $\Pi$ is constant and does not depend on the states.

We propose an estimation algorithm with different mode transition probabilities corresponding to different modes, called Mode-Dependent-HybridEstimation (MDHE) algorithm. Fig.1 shows a schematic of the MDHE algorithm. MDHE also uses a bank of Kalman filters (KF1 to KF4) to compute the mode probabilities $\mu_{i}(k+1)$ and the continuous state estimate $\hat{x}(k+1)$. However, individual Kalman fitters share information about the other Kalman fitters through new initial conditions at each time step. The components of MDHE in Fig.1 are described as follows: 


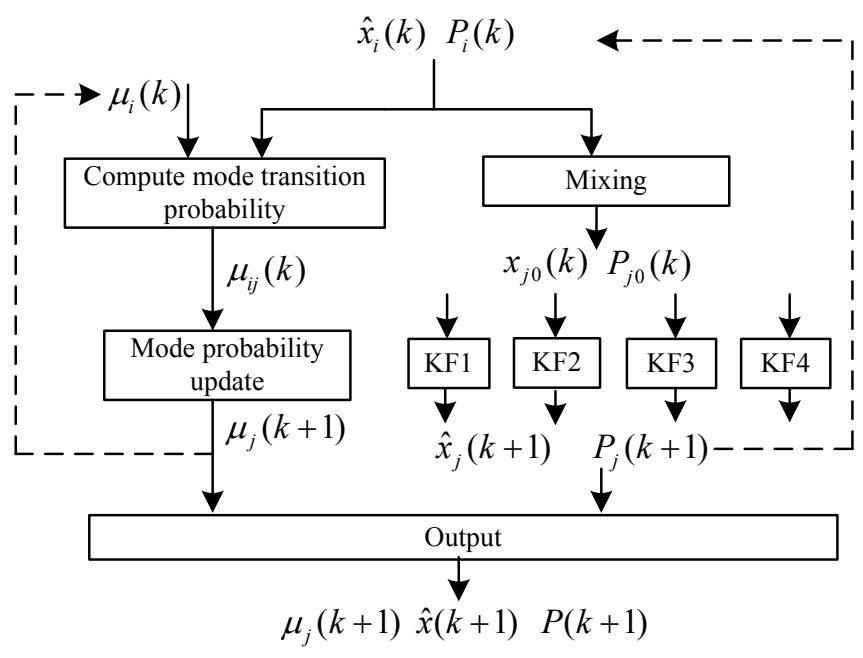

Figure 1: $\quad$ Structure of the MDHE algorithm.

1) Mixing probability. This is the probability that the system is in mode $i$ at time $k$, given that it is in mode $j$ at time $k+1(i, j \in\{1,2,3,4\})$

$$
\mu_{i j}(k+1 \mid k)=\frac{1}{c_{j}} \pi_{i j} \mu_{i}(k),
$$

where $c_{j}$ is a normalisation constant, $\mu_{i}(k)$ is a measure of the probability that the system is in mode $i$ at time $k$. It is assumed that $\mu_{i}(0)$ is given, which should be $\mu_{i}(0)=1$ for a specific mode $i$, with $\mu_{i}(0)=0$ for other modes.

2) New initial conditions. For each Kalman filters, the initial states $\hat{x}_{0 j}(k)$ and covariance $P_{0 j}(k)$ are computed by weighting the output of each Kalman filters with mixing probability as the weight

$$
\begin{gathered}
\hat{x}_{0 j}(k)=\sum_{i=1}^{N} \hat{x}_{i}(k) \mu_{i j}(k+1 \mid k) \\
P_{0 j}(k)=\sum_{i=1}^{N}\left[P_{i}(k)+\left[\hat{x}_{i}(k)-\hat{x}_{0 j}(k)\right] \times\left[\hat{x}_{i}(k)-\hat{x}_{0 j}(k)\right]^{T}\right] \mu_{i j}(k+1 \mid k)
\end{gathered}
$$

where $\hat{x}_{i}(k)$ and $P_{i}(k)$ are the estimation of state and its covariance of $\mathrm{KF} i$ at time $k$.

3) Mode Transition Probability. The mode transition matrix $\Pi$ is constant in the IMM algorithm. In this paper, we utilize the objective velocity-speed profile information to model the mode transition probabilities as modedependent probabilities. Each operation mode has a mode transition matrix and the system switches among these matrixes depending on the objective curve and continuous state. 
4) Kalman filter. Four Kalman filters run in parallel and each Kalman filter computes the $\hat{x}(k+1)$ and $P(k+1)$ using the initial conditions $\hat{x}_{0 j}(k)$ and $P_{0 j}(k)$.

5) Mode probabilities update. The probability of mode $j$ at time $k+1$ is computed as follow

$$
\mu_{j}(k+1)=\frac{1}{C} \Lambda_{j}(k+1) \sum_{i=1}^{N} \pi_{j i} \mu_{i}(k)
$$

where $C$ is a normalisation constant, $\Lambda_{j}(k+1)$ is the likelihood function, defined as

$$
\Lambda_{j}(k+1)=N_{p}\left(r_{j}(k+1) ; 0, S_{j}(k+1)\right)
$$

where $r_{j}(k+1)=z(k+1)-C_{j} \hat{x}_{j}(k+1 \mid k)$ is the residual of Kalman filter $j$, and $S_{j}(k+1)$ is its covariance.

6) Output. The estimation of state is a weighted sum of the estimates from four Kalman filters. The mode which has the highest mode probability is the mode estimate.

$$
\begin{gathered}
\hat{x}(k+1)=\sum_{j=1}^{N} \hat{x}_{j}(k+1) \mu_{j}(k) \\
P(k+1)=\sum_{j=1}^{N}\left\{P_{j}(k+1)+\left[\hat{x}_{j}(k+1)-\hat{x}(k+1)\right]\right\} \\
\left.\times\left[\hat{x}_{j}(k+1)-\hat{x}(k+1)\right]^{T}\right\} \mu_{j}(k+1) \\
\hat{m}(k+1)=\arg \max \mu_{j}(k+1)
\end{gathered}
$$

where $\hat{m}(k+1)$ is the mode estimation at time $k+1$.

\section{Simulations}

We consider an optimal speed-position trajectory of train's movement as shown in Fig.2.

The mode transition matrixes of MDHE are chosen as follows:

$$
\begin{aligned}
& \Pi_{\text {Power }}=\left[\begin{array}{llll}
0.9 & 0.06 & 0.03 & 0.01 \\
0.9 & 0.06 & 0.03 & 0.01 \\
0.9 & 0.06 & 0.03 & 0.01 \\
0.9 & 0.06 & 0.03 & 0.01
\end{array}\right], \quad \Pi_{\text {Hold }}=\left[\begin{array}{llll}
0.06 & 0.9 & 0.03 & 0.01 \\
0.06 & 0.9 & 0.03 & 0.01 \\
0.06 & 0.9 & 0.03 & 0.01 \\
0.06 & 0.9 & 0.03 & 0.01
\end{array}\right] \text {, } \\
& \Pi_{\text {Coast }}=\left[\begin{array}{llll}
0.01 & 0.06 & 0.9 & 0.03 \\
0.01 & 0.06 & 0.9 & 0.03 \\
0.01 & 0.06 & 0.9 & 0.03 \\
0.01 & 0.06 & 0.9 & 0.03
\end{array}\right], \quad \Pi_{\text {Coast }}=\left[\begin{array}{llll}
0.01 & 0.03 & 0.06 & 0.9 \\
0.01 & 0.03 & 0.06 & 0.9 \\
0.01 & 0.03 & 0.06 & 0.9 \\
0.01 & 0.03 & 0.06 & 0.9
\end{array}\right] \text {. }
\end{aligned}
$$


We compare the results of MDHE with that of IMM algorithm with constant mode transition matrix as

$$
\Pi_{I M M}=\left[\begin{array}{cccc}
0.9 & 0.1 / 3 & 0.1 / 3 & 0.1 / 3 \\
0.1 / 3 & 0.9 & 0.1 / 3 & 0.1 / 3 \\
0.1 / 3 & 0.1 / 3 & 0.9 & 0.1 / 3 \\
0.1 / 3 & 0.1 / 3 & 0.1 / 3 & 0.9
\end{array}\right] .
$$

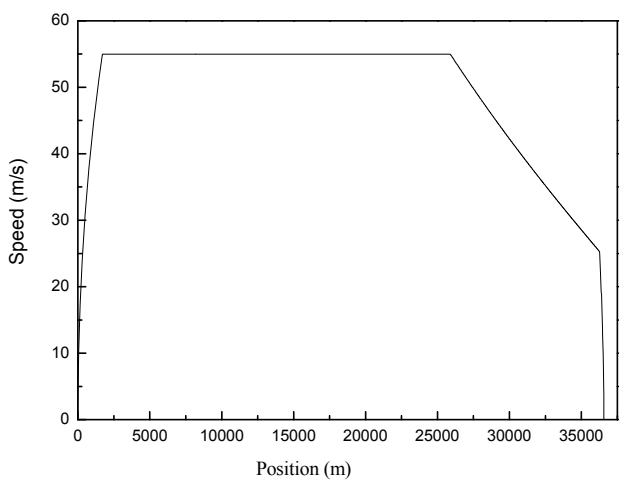

Figure 2: The optimal speed-position curve of the train.
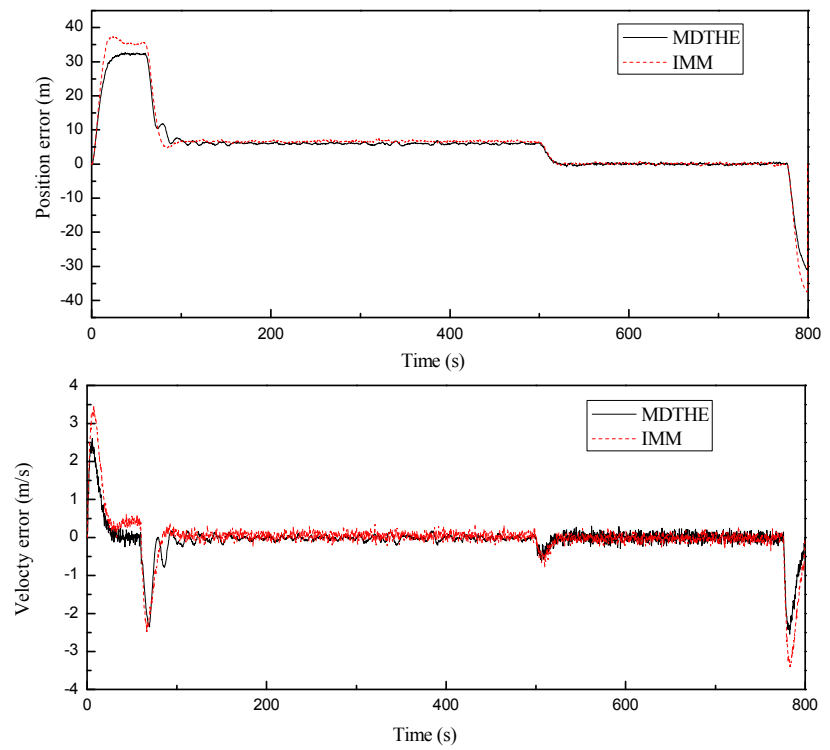

Figure 3: $\quad$ Comparison of tracking accuracy of MDTHE and IMM. 

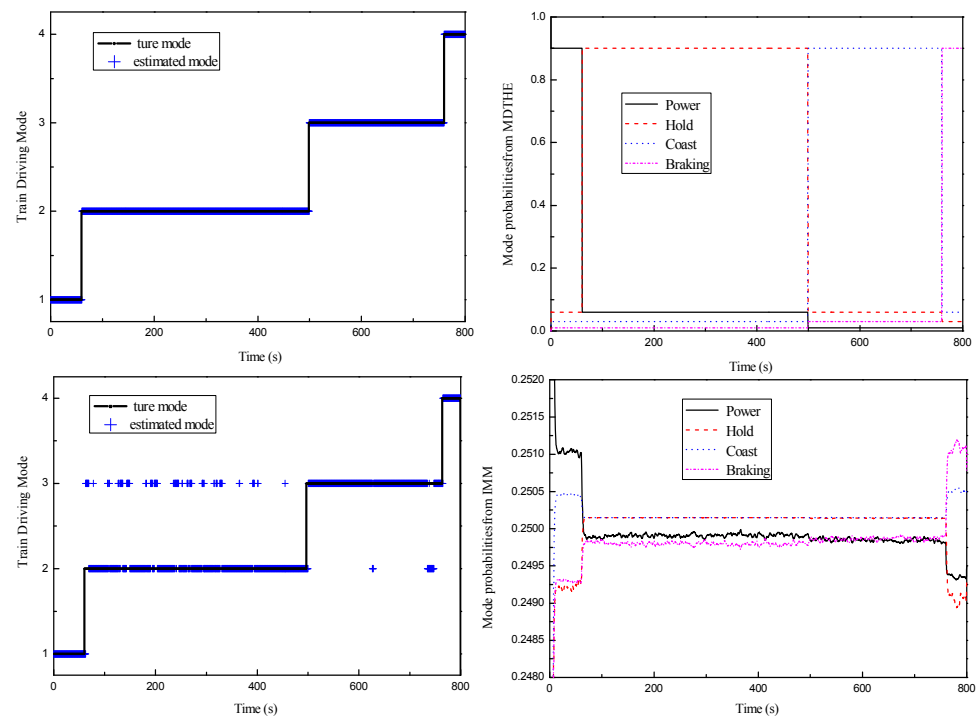

Figure 4: $\quad$ Estimation of modes and their probabilities.

Fig. 3 and Fig. 4 compare the tracking accuracy and the mode estimation accuracy of the algorithms. The tracking accuracy of MDHE and IMM algorithm depends on the design of the mode transition matrix. It is easy to see that MDHE has better tracking performance compared with IMM. The result also shows that the proposed algorithm improves the accuracy of the operation mode estimation.

\section{Conclusions}

In this paper, a hybrid system model is introduced to describe the train's dynamics. The stochastic factors during the train's movement are considered in this model. A new hybrid estimation algorithm is proposed for the train to track the objective velocity-position curve more accurately with mode dependent transition probability matrixes. Better tracking performance and the accuracy of the algorithm have been illustrated with simulations.

\section{Acknowledgements}

The authors would like to acknowledge that this work is supported by the Foundation No. 60634010, RCS2008ZQ003, and W08J0270.

\section{References}

[1] Seah, C.E. \& Hwang, I., Terminal-Area aircraft tracking using hybrid estimation [J]. Journal of Guidance, Control, and Dynamics, 32 (3), pp.83684, 2009. 
[2] Hwang, I., Balakrishnan H. \& Tomlin C., State estimation for hybrid systems: applications to aircraft tracking [C]. IEE Proceedings of Control Theory Application, 153(5), pp.556-566, 2006.

[3] Howlett, P.G. \& Pudney P.J., Energy-Efficient Train Control, Advances in Industrial Control, Springer, London, 1995.

[4] Zhu, J. \& Feng, X., The simulation research for the ATO model based on fuzzy predictive control, Autonomous Decentralized Systems, ISADS Proceedings. 2005.

[5] Blom H.A.P. \& Bar-Shalom Y., The interacting multiple model algorithm for systems with markovian switching coefficients. IEEE Transactions on automatic control, 33(8), pp.780-783, 1988.

[6] Khmelnitsky E., On an optimal control problem train operation, IEEE Transactions on Automatic Control, 45(7), pp.1257-1266, 2000. 